\title{
Correction to: Emergence of robust anthropogenic increase of heat stress-related variables projected from CORDEX-CORE climate simulations
}

\author{
Eun-Soon $\mathrm{Im}^{1,2}\left(\mathbb{D} \cdot\right.$ Nguyen-Xuan Thanh ${ }^{2} \cdot$ Liying Qiu $^{1} \cdot$ Moetasim Ashfaq $^{3} \cdot$ Xuejie Gao $^{4} \cdot$ Tong Yao $^{4} \cdot$ Csaba Torma $^{5}$. \\ Mojisola O. Adeniyi ${ }^{6} \cdot$ Sushant Das $^{7} \cdot$ Graziano Giuliani $^{7} \cdot$ Erika Coppola $^{7} \cdot$ Filippo Giorgi $^{7}$
}

Published online: 9 March 2021

(c) Springer-Verlag GmbH Germany, part of Springer Nature 2021

\section{Correction to: Climate Dynamics \\ https://doi.org/10.1007/s00382-020-05398-w}

The original version of the article contained error in the Fig. 8 caption was swapped and below is the corrected caption.

Fig. 8 TOE patterns of temperature $(\mathbf{a}, \mathbf{c}, \mathbf{e})$ and $\mathrm{Tw}(\mathbf{b}$, $\mathbf{d}, \mathbf{f})$ as a function of elevation over the European $(\mathbf{a}, \mathbf{b})$, East Asia $(\mathbf{c}, \mathbf{d})$, and Africa $(\mathbf{e}, \mathbf{f})$ domains derived from the RCMs and GCMs simulations. The different size of circle is proportional to the percentage of number of grids included in individual height ranges. The total number of grids used for the calculation of RCMs and GCMs are different because their resolutions are different.

The original article has been corrected.

The original article can be found online at https://doi.org/10.1007/ s00382-020-05398-w.

Eun-Soon Im

ceim@ust.hk

1 Department of Civil and Environmental Engineering, The Hong Kong University of Science and Technology, Academic Building 3594, Clear Water Bay, Kowloon, Hong Kong, China

2 Division of Environment and Sustainability, The Hong Kong University of Science and Technology, Hong Kong, China

3 Computational Sciences and Engineering Division, Oak Ridge National Laboratory, Oak Ridge, TN, USA

4 Climate Change Research Center, Institute of Atmospheric Physics, Chinese Academy of Sciences, Beijing, China

5 Department of Meteorology, Eötvös Loránd University, Budapest, Hungary

6 Department of Physics, University of Ibadan, Ibadan, Nigeria

7 Earth System Physics, Abdus Salam International Centre for Theoretical Physics, Trieste, Italy 